\title{
Detection of Filifactor alocis and Dialister pneumosintes in Biofilms of Smokers and Nonsmokers with Chronic Periodontitis: A PCR Study
}

\author{
Shwetha Eshwarappa ${ }^{1}$, Chethana K Chidambar ${ }^{2}$, Shrinidhi M Shankar ${ }^{3}$
}

\begin{abstract}
Aim and objective: Detection of Filifactor alocis and Dialister pneumosintes in smokers and nonsmokers with chronic generalized periodontitis. Materials and methods: Out of 60 patients diagnosed with chronic generalized periodontitis, 30 current smokers and 30 nonsmokers were recruited for the study. Clinical parameters such as gingival bleeding index, plaque index, and probing pocket depth were recorded. Subgingival plaque samples were collected and subjected to a multiplex polymerase chain reaction (PCR) assay.

Results: Collected plaque samples of nonsmokers with chronic periodontitis patients showed a slight increase in numbers of $F$. alocis and $D$. pneumosintes species when compared with smokers, although statistically not significant.

Conclusion: The distribution of $F$. alocis and $D$. pneumosintes which are considered as the masked pathogens of the periodontal disease was not significantly different in nonsmokers and smokers with chronic periodontitis.

Clinical significance: These species which were rarely studied can be considered as markers for periodontal disease, as they denote increased periodontal destruction.

Keywords: Biofilm, Dialister pneumosintes, Filifactor alocis, Polymerase chain reaction, Smokers.

World Journal of Dentistry (2021): 10.5005/jp-journals-10015-1846
\end{abstract}

\section{INTRODUCTION}

Periodontal diseases are a result of specific microbial complexes in subgingival biofilms. ${ }^{1}$ Polymicrobial associations which exhibit structural and functional heterogeneity are termed biofilms. Periodontitis is a dysbiotic disease wherein there is a shift in the microbial profile from a gram-positive toward gram-negative ${ }^{2}$ or more from orange to red complex, thus gradually changing the symbiotic host-microbe relationship to a more pathogenic one. Contrary to this microbial association with the disease, recent studies have proven the association of other organisms like Veillonella species. However, these pathogens have not been isolated or proven to have a direct role in the etiology of periodontitis. ${ }^{3}$ Some of the new organisms associated with periodontal disease are Filifactor alocis and Dialister pneumosintes.

Filifactor alocis a fastidious, gram-positive, obligatory anaerobic rod that possesses trypsin-like enzymatic activity similar to Porphyromonas gingivalis and Treponema denticola. In comparison to the other traditional periodontal pathogens, the high incidence of F. alocis in the periodontal pocket compared with its absence in healthy or periodontitis-resistant patients has highlighted its importance in the infectious disease process. It has also been identified as one of the prevalent phylotypes in cases of failed endodontic treatment and associated with peri-implantitis. Filifactor alocis has a unique property such as resistance to oxidative stress and genes coding for a well-developed amino acid metabolic pathway, which can allow it to colonize and polymicrobial synergistic relationship which can enhance its invasive capacity and survive with other traditional periodontal pathogens in the stress environment of the periodontal pocket. Filifactor alocis was detected in elevated numbers in chronic periodontitis patients and was therefore proposed as a potential marker for active disease. ${ }^{4}$
${ }^{1-3}$ Department of Periodontics, Sharavathi Dental College and Hospital, Shivamogga, Karnataka, India

Corresponding Author: Chethana K Chidambar, Department of Periodontics, Sharavathi Dental College and Hospital, Shivamogga, Karnataka, India, Phone: +91 9448168733, e-mail: anuhgd@gmail.com How to cite this article: Eshwarappa S, Chidambar CK, SM Shankar. Detection of Filifactor alocis and Dialister pneumosintes in Biofilms of Smokers and Nonsmokers with Chronic Periodontitis: A PCR Study. World J Dent 2021;12(4):301-305.

Source of support: Nil

Conflict of interest: None

Dialister pneumosintes, a resident of the upper respiratory tract and oral cavity, is a small, non-fermentative, gram-negative anaerobic rod, which has been isolated from periodontal lesions and endodontic infections. It is often associated with other bacterial pathogens and viruses in mixed periodontal and root canal infections. ${ }^{1}$

Chronic periodontitis results from the presence of complex microbial communities in the subgingival sulcus influenced by smoking, a major environmental factor associated with the development of extensive and severe periodontal disease. One of the first ecosystems to come into contact with tobacco smoke, and to be affected by it, is the oral microbiome. Smokers present a highat-risk cohort for periodontitis, a microbially driven oral disease that affects two-thirds of adult humans. Smokers acquire these pathogens within 24 hours of biofilm formation and are associated with pathogen enrichment. ${ }^{5}$

The present study aimed to detect the presence of F. alocis and $D$. pneumosintes in the subgingival plaque of smokers and

() Jaypee Brothers Medical Publishers. 2021 Open Access This article is distributed under the terms of the Creative Commons Attribution 4.0 International License (https://creativecommons.org/licenses/by-nc/4.0/), which permits unrestricted use, distribution, and non-commercial reproduction in any medium, provided you give appropriate credit to the original author(s) and the source, provide a link to the Creative Commons license, and indicate if changes were made. The Creative Commons Public Domain Dedication waiver (http://creativecommons.org/publicdomain/zero/1.0/) applies to the data made available in this article, unless otherwise stated. 
nonsmokers diagnosed with chronic periodontitis by using the polymerase chain reaction (PCR) technique.

\section{Materials and Methods}

A total of 60 patients who required periodontal treatment were recruited from the Outpatient Department of Periodontology, Sharavathi Dental College and Hospital, Shivamogga. Patients diagnosed with chronic generalized periodontitis as per the AAP 1999 criteria were grouped into 30 current smokers ${ }^{6}$ and 30 nonsmokers. The study was conducted in full accordance with ethical principles and was approved by the institutional ethics committee (Ref no:-SDC/SMG/2017/649) and also written informed consent was obtained from each patient. Patients in the age group of 35-55 years with at least 20 natural teeth and a minimum of 6 teeth with periodontal pockets with a depth of $\geq 4 \mathrm{~mm}$ were included. ${ }^{7}$ Exclusion criteria included patients who had a history of antibiotic use or periodontal treatment 6 months before the study or with systemic conditions known to affect the periodontal status, immuno-compromised individuals, pregnant, and lactating women. Clinical examination was done by a single examiner using William's periodontal probe. Plaque index, ${ }^{8}$ gingival bleeding index, ${ }^{9}$ and probing pocket depth (PPD) were recorded. The patients were divided into two groups; Group I comprised 30 nonsmokers while group II comprised 30 current smokers. This study was cross-sectional and observational in nature, hence no controls were included.

\section{Method of Collection of Sample and Microbial Analysis}

The subgingival plaque samples were collected before the initial phase of periodontal therapy. The samples were collected by inserting a sterile curette into the periodontal pockets. Later, it was pooled in $50 \mu \mathrm{L}$ TE transport medium (Tris-HCl, Tris-EDTA, and distilled water) and the samples were sent to the laboratory for PCR analysis. Multiplex conventional PCR was carried out for the detection of $D$. pneumosintes and $F$. alocis by using DNA extracts (Template) PCR master mix (Ampliqon red) and species-specific PCR primers such as forward primer: CAG GTG GTT TAA CAA GTT AGT GG [Bioserve Biotechnologies (India) Pvt Ltd, Hyderabad]and reverse primer: CTA AGT TGT CCT TAG CTG TCT CG with base pair of 594 for $F$. alocis and forward primer: TTC TAA GCA TCG CAT GGT GC [Bioserve Biotechnologies (India) Pvt Ltd, Hyderabad] and reverse primer: GAT TTC GCT TCT CTT TGT TG with base pair of 1,105 for D. pneumosintes. Initial denaturation was done at $950^{\circ} \mathrm{C}$ for 5 minutes followed by 36 cycles of denaturation at $940^{\circ} \mathrm{C}$ for 30 seconds, annealing at $550^{\circ} \mathrm{C}$ for 1 minute, and extension at $720^{\circ} \mathrm{C}$ for 2 minutes. The final extension was carried out at $720^{\circ} \mathrm{C}$ for 5 minutes. Post-PCR analysis was done by running the amplified products on $2 \%$ agarose gel electrophoresis. The PCR products were loaded into $2 \%$ agarose gel matrix and electrophoresis was run at $75 \mathrm{~V}$ for 2 hours. The gel was stained with ethidium bromide and observed under a UV transilluminator. Photos were captured using the gel doc system. The bands obtained for each sample were compared with the DNA ladder and a specific band of organism was identified (Fig. 1). ${ }^{10,11}$

\section{Statistical Analysis}

The results obtained from various parameters were subjected to statistical analysis. Results were expressed as mean with standard deviation. Intergroup comparisons were done by using the

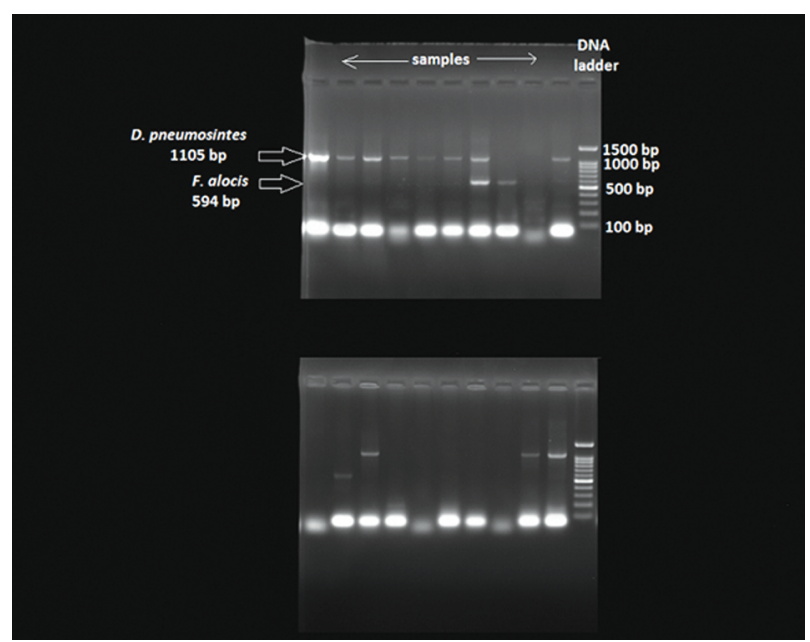

Fig. 1: Representative PCR amplicons. Clinical samples are positive for the presence of bacteria after amplification by multiplex PCR for Filifactor alocis and Dialister pneumosintes. Clinical samples are positive for $D$. pneumosintes. (Lane 1,7) Clinical samples are positive for F. alocis. (Lane 7) Clinical samples are positive for both species. (Lane 7)

Chi-square test and independent $t$-test. For all the tests, a $p$ value of 0.05 or less was considered for statistical significance.

\section{Results}

On gender distribution, 24 were males and 6 were females in group I whereas 27 were males and 3 were females in group II. No difference was seen with the Chi-square with Yates's correction = $0.5231, p=0.4701$ (Table 1).

The mean age in subjects with smokers was 47.43 with a standard deviation of 4.68 and in subjects with nonsmokers were 45.93 with a standard deviation of 6.00. No significant difference was observed between group I and group II with mean age ( $t=$ 1.0797, $p=0.2848$; Table 1).

The mean plaque index score in subjects with smokers was 1.60 with a standard deviation of 0.22 and in subjects with nonsmokers was 1.51 with a standard deviation of 0.21 . No significant difference was observed between group I and group II with mean plaque index scores ( $t=1.6798, p=0.0984$; Table 2$)$.

The mean gingival bleeding index score in subjects with smokers was 0.65 with a standard deviation of 0.14 and in subjects with nonsmokers was 0.35 with a standard deviation of 0.11 significant difference was observed between group I and group II with mean gingival bleeding scores $(t=9.4126, p=0.0001)$. The mean gingival bleeding index was significantly higher in group I as compared to group II ( $p=0.0001$ and Table 3).

The mean probing depth score in subjects with smokers was 6.11 with a standard deviation of 0.60 and in subjects with nonsmokers were 6.07 with a standard deviation of 0.68 . No significant difference was observed between group I and group II with mean probing depth scores $(t=0.2671, p=0.7904)$. The mean probing depth scores were similar in group I and group II (Table 4).

In the present study, the presence of $D$. pneumosintes and $F$. alocis was determined by multiplex PCR amplification of reference genomic DNA. Primer pairs were used for the identification of two pathogens. Clinical samples that were positive for F. alocis and/or $D$. pneumosintes also showed one band of the predicted size per species and the absence of non-specific amplification products. 
Table 1: Comparison of nonsmoker and smoker with mean age by independent $t$-test and distribution of male and females in nonsmoker and smoker

\begin{tabular}{|c|c|c|c|c|c|c|c|c|}
\hline \multirow[b]{2}{*}{ Groups } & \multicolumn{4}{|c|}{ Age } & \multicolumn{4}{|c|}{ Sex } \\
\hline & Mean & $S D$ & $S E$ & $p$ value ${ }^{*}$ & Male & Female & Total & $p$ value* \\
\hline Nonsmoker & 47.43 & 4.68 & 0.85 & 0.2848 & 24 & 6 & 30 & 0.4701 \\
\hline Smoker & 45.93 & 6.00 & 1.10 & & 27 & 3 & 30 & \\
\hline
\end{tabular}

$\mathrm{SD}$, standard deviation; $\mathrm{SE}$, standard error

*The mean difference is significant at the 0.05 level; NS, not significant

Table 2: Comparison of nonsmoker and smoker with plaque index scores by independent $t$-test

\begin{tabular}{llllll}
\hline Groups & Mean & $S D$ & $S E$ & t value & $p$ value \\
\hline Nonsmoker & 1.60 & 0.22 & 0.04 & 1.6798 & 0.0984 \\
Smoker & 1.51 & 0.21 & 0.04 & & \\
\hline
\end{tabular}

SD, standard deviation; SE, standard error

The mean difference is significant at the 0.05 level

Table 3: Comparison of nonsmoker and smoker with gingival bleeding index scores by independent $t$-test

\begin{tabular}{llllll}
\hline Groups & Mean & $S D$ & $S E$ & t value & $p$ value \\
\hline Nonsmoker & 0.65 & 0.14 & 0.03 & 9.4126 & $0.0001^{*}$ \\
Smoker & 0.35 & 0.11 & 0.02 & & \\
\hline
\end{tabular}

$\mathrm{SD}$, standard deviation; SE standard error

${ }^{*} p<0.05$ indicates significant between them

Table 4: Comparison of nonsmoker and smoker with probing depth scores by independent $t$-test

\begin{tabular}{llllll}
\hline Groups & Mean & $S D$ & $S E$ & t value & $p$ value \\
\hline Nonsmoker & 6.11 & 0.60 & 0.11 & 0.2671 & 0.7904 \\
Smoker & 6.07 & 0.68 & 0.12 & & \\
\hline
\end{tabular}

$\mathrm{SD}$, standard deviation; $\mathrm{SE}$, standard error

The mean difference is significant at the 0.05 level

Table 5: Comparison of nonsmoker and smoker with presence status of Dialister pneumosintes

\begin{tabular}{llllll}
\hline Dialister pneumosintes & Nonsmoker & $\%$ & Smoker & $\%$ & Total \\
\hline Absent & 12 & 40.00 & 19 & 63.33 & 31 \\
Present & 18 & 60.00 & 11 & 36.67 & 29 \\
Total & 30 & 100.00 & 30 & 100.00 & 60 \\
\hline
\end{tabular}

Chi-square $=3.2701 p=0.0711$

The mean difference is significant at the 0.05 level

Table 6: Comparison of nonsmoker and smoker with presence status of Filifactor alocis

\begin{tabular}{lllccc}
\hline Filifactor alocis & Nonsmoker & $\%$ & Smoker & $\%$ & Total \\
\hline Absent & 16 & 53.33 & 21 & 70.00 & 37 \\
Present & 14 & 46.67 & 9 & 30.00 & 23 \\
Total & 30 & 100.00 & 30 & 100.00 & 60 \\
\hline
\end{tabular}

Chi-square $=1.7636 p=0.1846$

The mean difference is significant at the 0.05 level

The DNA mixture of nonsmokers (8 samples) and smokers (15 samples) lacking DNA of the two target species yielded negative results in the multiplex $P C R$.

Dialister pneumosintes was identified in $60 \%$ (18 samples were positive) from nonsmokers subjects and $36.6 \%$ (11 samples were positive) from smokers out of the 30 samples. The prevalence of the organism was highest in the nonsmoker group when compared to the smoker group by using the Chi-square test with the value of 1.7636 and $p$ value of 0.1846 , which is not significant statistically (Table 5).
Considering all the collected samples, F. alocis was identified in $46.67 \%$ (14 samples were positive) from nonsmokers subjects and $30 \%$ (9 samples were positive) from smokers out of the 30 samples. The prevalence of the organism was highest in group I when compared to group $B$ by using the Chi-square test with the value of 1.7636 and $p$ value of 0.1846 . While detection frequencies did not differ significantly between nonsmokers and smokers (Table 6); however, D. pneumosintes was seen more in numbers on intra comparison between the organisms in both the groups (Fig. 2). 


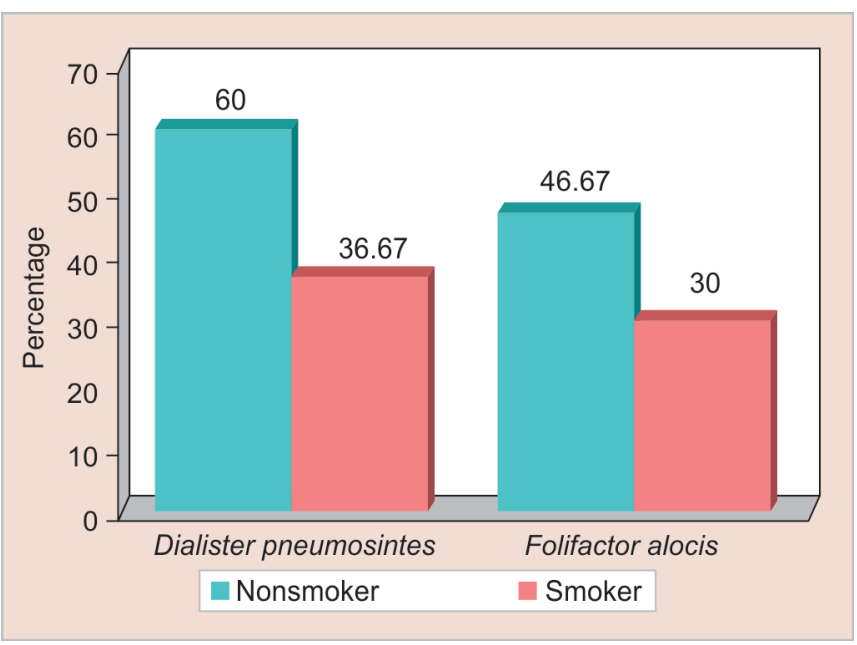

Fig. 2: Comparison of nonsmoker and smoker with the presence of Filifactor alocis and Dialister pneumosintes by independent $t$-test

Clinically, subgingival plaque of participants with periodontitis has shown a higher prevalence of $D$. pneumosintes and F. alocis in the nonsmoker group rather than the smokers.

\section{Discussion}

Periodontal diseases are classified as complex disorders where environmental, lifestyle, and genetic factors contribute to their etiology. The most important environmental factor is the diverse microbiota arranged in a biofilm, which acts as an initiator of periodontal destruction. Also, lifestyle factors such as stress, smoking, and oral hygiene habits act as modifiers of disease progression. Smoking is responsible for accelerated periodontal destruction and increased risk of periodontitis in younger adults, as an anaerobic environment promotes the growth of gram-negative periodontal pathogens in the subgingival plaque. However, on the contrary, a study using Gram staining techniques failed to show a significant difference in the subgingival microflora between smokers and nonsmokers. ${ }^{12}$ Hence, a study done by Qiu et al. $^{13}$ has suggested that smoking may affect the host response.

The present study used a multiplex conventional PCR protocol for simultaneous detection of $D$. pneumosintes and $F$. alocis. Multiplex conventional PCR allows the simultaneous detection of different bacterial species. This PCR assay showed high specificity because only DNA mixtures containing DNA from both species generated the expected amplicons (one per species) after multiplex PCR amplification. This gave the clinical samples positive or negative results for either $D$. pneumosintes and $F$. alocis or both. Positive cases showed only the species-specific bands and the absence of non-specific bands.

In the present study, the presence of F.alocis and D. pneumosintes in smokers were 30 and $36.61 \%$, whereas in nonsmokers were 46.67 and $60 \%$, respectively. Chronic periodontitis is a source of oxidative stress, leading to elevated numbers of $F$. alocis in these environments. The virulence factors of $F$. alocis allow it to persist in extreme oxidative stress situations. ${ }^{14}$ Furthermore, smoking changes the subgingival bacterial profile by reducing beneficial species and increase of treponemes, ${ }^{12}$ and also has a direct inhibitory effect on the host response. ${ }^{13}$ It increases the oxidative stress quotient within the subgingival environment making it conducive for the growth of F. alocis. However, in the present study, on the contrary, there was an increase in the number of both the organisms in nonsmokers with chronic periodontitis rather than smokers with chronic periodontitis.

These organisms are also associated with compromised pulp status, ${ }^{10}$ patients were reassessed for the pulp status of periodontally involved teeth using an electric pulp test. Consequently, 30\% of teeth showed altered pulpal response suggestive of a developing endo lesion. Hence, the above findings substantiate the presence of endodontic pathogens in a periodontal pocket, even if the lesion is not a part of perio-endo continuum. ${ }^{15}$

Filifactor alocis as well as D. pneumosintes have been known to be endodontic pathogens. A study done by Siqueira and Rocas ${ }^{10}$ revealed more number of $D$. pneumosintes (50\%) than $F$. alocis $(40 \%)$ in endodontic infections. Similar results were found in the present study. The number of $D$. pneumosintes was found more in subgingival samples irrespective of smokers and nonsmokers when compared to the number of $F$. alocis.

Research is limited about the pathogenicity of F. alocis. Filifactor alocis produces FACIN a complement inhibiting substance similar to a dysbiotic infection caused by a keystone pathogen that alters the subgingival environment. This change leads to a complement lead inflammation where F. alocis inhibits all pathways and causes periodontal disease. A study done by Jansson et al. in the mid-90s have explored the role of endodontic pathogens in the progression of marginal tissue breakdown. ${ }^{15}$

The prominent presence of $D$. pneumosintes in the subgingival microbiota of periodontal disease has come to light only in the recent past. This microbe-associated frequently with endodontic infections, is also found to be associated with subgingival microbiota of aggressive periodontitis (AgP) patients. ${ }^{10}$ Earlier study done by Gomes et al. have shown D. pneumosintes and F. alocis were detected in higher numbers in deep pockets of smokers. ${ }^{16}$ However, data are limited about the virulence action of $D$. pneumosintes. The subgingival occurrence of $D$. pneumosintes might have been underestimated because of its strict anaerobic requirement and its slow growth. The high prevalence of $D$. pneumosintes in advanced periodontitis lesions and the apparent pathogenicity of the organism places it in the group of suspected periodontal pathogens.

The higher numbers of $F$. alocis and $D$. pneumosintes in $C P$ nonsmokers than in CP smokers are of concern as it could be a passive increase or could have an actual contribution to the pathogenesis of the periodontal disease. This clinical significance could be attributed to the presence of undiagnosed endodontic lesions or teeth which needed endodontic attention along with the periodontal disease. Filifactor alocis is known to colonize polymicrobial biofilm communities. An in vitro community model showed the synergistic association of $F$. alocis and Fusobacterium nucleatum, whereas Streptococcus gordonii was an antagonist to the accumulation of F. alocis. ${ }^{17}$

Collectively, these observations showed that the pattern of F. alocis colonization will depend on the spatial composition of the microbial microenvironment. Filifactor alocis preferentially accumulates at the site rich in Fusobacterium nucleatum. On the other hand, presence of Streptococcus gordonii, its aggregation, and multiplication could have contributed to a decrease in the number of $F$. alocis in the CP smokers group. ${ }^{18}$ This complex synergistic and antagonistic interaction which occurs within the oral microbial community result in the success or failure of microbial colonization. Hence, colonization of $F$. alocis heavily depends on the inhabitant 
of the microbial community. A study of pathogenicity, virulence factor, and colonization pattern of $F$. alocis and $D$. pneumosintes with other microbial communities is further required. The limited sample size and quantitative analysis could have been attributed to statistical insignificance in this study.

The present study evaluated an association between the presence of these microbes and periodontitis in smokers and nonsmokers and assessed whether to consider them as markers of periodontal disease. However, within the limitations of sample size, culture methods, and interventional trials, it can only be concluded that higher proportions of these novel organisms are associated with the progression of periodontal destruction and smoking contributes only as a risk factor and no role as a causative agent.

\section{Conclusion}

In the present study, even though there was no statistically significant difference between the groups, considering the limited sample size $F$. alocis and D. pneumosintes were noted in higher numbers in nonsmokers compared with smokers with chronic periodontitis. Hence, the use of these organisms as markers of periodontal disease with smokers should be considered with discretion. However, a higher presence of these masked pathogens could be considered as one of the contributing factors in periodontal disease pathogenesis and progression.

\section{References}

1. Ferraro CTL, Gornic C, Barbosa AS, et al. Detection of Dialister pneumosintes in the subgingival biofilm of subjects with periodontal disease. Anerobe 2007;13(5-6):244-248. DOI: 10.1016/j. anaerobe.2007.09.002.

2. Shaikh HF, Patil SH, Pangam TS, et al. Polymicrobial synergy and dysbiosis: an overview. J Indian Soc Periodontol 2018;22(2):101. DOI: 10.4103/jisp.jisp_385_17.

3. Berezow AP, Darveau RP. Microbial shift and periodontitis. Periodontol 2000 2011;55(1):36-47. DOI: 10.1111/j.1600-0757.2010.00350.x.

4. Schlafer S, Riep B, Griffen AL, et al. Filifactor alocis-involvement in periodontal biofilms. BMC Microbiol 2010;10(1):66. DOI: 10.1186/14712180-10-66.

5. Mason MR, Preshaw PM, Nagaraja HN, et al. The subgingival microbiome of clinically healthy current and never smokers. ISME J 2015;9(1):268. DOI: 10.1038/ismej.2014.114.
6. Newman M, Takei HH, Klokkevold PR, et al. Carranza's clinical periodontology. 10th ed., St. Louis, Mo: Elsevier Saunders; 2006. 14: Smoking and Periodontal Disease pp. 251-258.

7. Armitage GC. Development of a classification system for periodontal diseases and conditions. Ann Periodontol 1999;4(1):1-6. DOI: 10.1902/ annals.1999.4.1.1.

8. Silness J, Loe H. Periodontal disease in pregnancy. II. Correlation between oral hygiene and periodontal condition. Acta Odontol Scand 1964;22(1):121-135. DOI: 10.3109/00016356408993968.

9. Loe $\mathrm{H}$. The gingival index, the plaque index and the retention index systems. J Periodontol 1967;38(6 Part II):610-616. DOI: 10.1902/ jop.1967.38.6 part2.610.

10. Siqueira Jr JF, Rocas IN. Simultaneous detection of Dialister pneumosintes and Filifactor alocis in endodontic infections by $16 \mathrm{~S}$ rDNA-directed multiplex PCR. J Endod 2004;30(12):851-854. DOI: 10.1097/01.DON.0000132300.13023.5D.

11. Vanpelt E, Belkum VAV, Hays JP. Principles and technical aspects of PCR amplification. Springer; 2008. p. 34.

12. Karasneh JA, Al Habashneh RA, Marzouka NA, et al. Effect of cigarette smoking on subgingival bacteria in healthy subjects and patients with chronic periodontitis. BMC Oral Health 2017;17(1):64. DOI: 10.1186/s12903-017-0359-4.

13. Qiu F, Liang CL, Liu H, et al. Impacts of cigarette smoking on immune responsiveness: Up and down or upside down? Oncotarget 2017;8(1):268. DOI: 10.18632/oncotarget.13613.

14. Aruni AW, Roy F, Fletcher HM. Filifactor alocis has virulence attributes that can enhance its persistence under oxidative stress conditions and mediate invasion of epithelial cells by Porphyromonas gingivalis. Infect Immun 2011;79(10):3872-3886. DOI: 10.1128/IAI. 05631-11.

15. Jansson L, Ehnevid H, Blomlöf $L$, et al. Endodontic pathogens in periodontal disease augmentation. J Clin Periodontol 1995;22(8):598602. DOI: 10.1111/j.1600-051x.1995.tb00811.x.

16. Gomes SC, Piccinin FB, Oppermann RV, et al. Periodontal status in smokers and never-smokers: Clinical findings and real-time polymerase chain reaction quantification of putative periodontal pathogens. J Periodontol 2006;77(9):1483-1490. DOI: 10.1902/ jop.2006.060026.

17. Wang $\mathrm{Q}$, Wright $\mathrm{C}$, Dingming $\mathrm{H}$, et al. Oral community interactions of Filifactor alocis in vitro. PLoS ONE 2013;8(10):e76271. DOI: 10.1371/ journal.pone.0076271.

18. Huang R, Li M, Ye M, et al. Effects of nicotine on Streptococcus gordonii growth, biofilm formation, and cell aggregation. Appl Environ Microbiol 2014;80(23):7212-7218. DOI: 10.1128/AEM. 02395-14. 